Karmen Teržan Kopecky ${ }^{*}$

Universität Maribor
UDK $81: 316.77$

DOI: 10.4312/linguistica.59.1.333-347

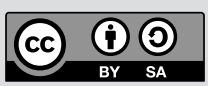

\title{
DIE ENTITÄT INFORMATION IM SPANNUNGSFELD ZWISCHEN WORT UND TEXT
}

\section{ALLGEMEINES ZUR ENTITÄT INFORMATION}

Informationen werden von vielen unterschiedlichen Wissenschaften definiert. Sie dienen der Vergrößerung oder Explizierung des Wissens und/oder bilden Ursachen für Vorgänge. Keine der Bestimmungen des Begriffs Information widerspricht der Auffassung, dass sich uns Informationen als Zeichen präsentieren. Nachstehend werden einige der relevantesten Erkenntnisse sowohl aus dem Bereich der Naturwissenschaften als auch aus dem Bereich der Sozial- und Geisteswissenschaften zusammenfassend dargelegt, bis schließlich auf sprachlich (durch Silbe, Wort, Satz, Text, Diskurs) kodierte Informationen eingegangen wird, von denen unsere sprachliche Kommunikation getragen ist.

\subsection{Naturwissenschaftliche Definitionen}

Die Mikrowissenschaften z. B. Quantenphysik, Mikrobiologie, Nanowissenschaften usw., stellt Oktabetz 2014 in seinem Buch Das Infoversum fest, haben neue Wissensbereiche bereitgestellt, durch die das Verstehen der Welt durch den Menschen immer schärfere Konturen gewinnt, da sie Einblick in die kleinsten Mikroteilchen ermöglichen. Die Beschaffenheit und der Transport von Informationen werden als Grundlagen der Wirklichkeit vorausgesetzt, verantwortlich für die Beschleunigung des Erwerbs und der Verbreitung des Wissens sowie die exponentiell wachsende Erkenntniserweiterung.

Es wird davon ausgegangen, dass die Information als die Basisentität sich weder als Materie noch als Energie beschreiben lässt (vgl. Wiener 1948), denn sie ist von einer Vielzahl biochemischer Vorgänge getragen und beruht auf dem Interagieren der kleinsten bislang bekannten Teilchen, der Quanten und Quarks, der Nano-Teilchen, Photonen, Gravitonen usw., von welchen die beiden Erstgenannten einfache digitale Befehle erzeugen und sich jenseits von Raum und Zeit miteinander verschränken und derart kodierte Informationen über ungeahnte Weiten transportieren können.

Die oben dargelegten Erkenntnisse lassen die Existenz von Informationen auch außerhalb unserer Raumzeitlichkeit vermuten, d. h. dass sich die apriori bestehenden, vom Erzeuger und Adressaten unabhängigen Informationen, sobald sie „zu Wort werden", den unterschiedlichsten Facetten ihrer Umwelt anpassen müssen, um für konkrete Zweckbestimmungen bereit zu stehen.

karmen.kopecky@gmail.com 
Durch moderne Messgeräte und -techniken sind, wie bereits oben kurz geschildert, immer kleiner werdende Zeit- und Raumeinheiten zum wichtigen Bestandteil unseres Weltbildes geworden. Sogar der Begriff des Augenblicks, mit dem in der Linguistik der Begriff der eigentlichen Gegenwart (hic-et-nunc-Präsens) definiert wird, kann mit immer kleineren Maßeinheiten gemessen werden (z. B.: von Attosekunden (trillionstel Sekunden) (vgl. Oktabetz 2014.) Die elementare Informationseinheit wäre, so die Informationstechnik auf der Basis der Quantentheorie, der Quantenbit. Es handelt sich dabei um Zeit- bzw. Informationseinheiten, die sich jenseits unserer Vorstellungskraft bewegen und natürlich die Begriffswelt des zeitlichen Verlaufs und der Beschaffenheit des Raumes enorm beeinflussen. Die Zeitspanne der als Gegenwart empfundenen Informationen könnte demzufolge dehnbarer (größer) oder aber noch wesentlich kleiner sein, als unsere Vorstellung dies traditionell angenommen hatte. Noch wesentlich größer ist der Raum von gespeicherten und aus dem Gedächtnis abrufbaren Informationen (Vergangenheit) und der erwarteten/ zukünftigen Informationen, deren Begrenzung noch kleiner ist als bei Informationen, die der Vergangenheit angehören. Eine Folge davon ist eine immer größer werdende Genauigkeit der Interpretation von grundlegenden kognitiven Entitäten wie Raum und Zeit.

Die Mikrobiologie, genauer die Zellforschung untersucht Prozesse der Informationenspeicherung in Zellen: Diese erfolgt unter Zusammenwirkung organischer und anorganischer Stoffe als Informationsträger und-verarbeiter (z. B. Nano-Bio-Chips) und schließt grundlegende Informationen der Gattung Mensch sowie auch jedes einzelnen Individuums ein. In Berücksichtigung dieser Erkenntnisse geht man davon aus, dass Lebewesen Produkte von Elementarinformationen sind, denn auch die im Erbmaterial enthaltenen Eigenschaften sind Informationen. Laut neuester Forschungsergebnisse in diesem Bereich kann der Einsatz von Bakterien als Datenträgern zu medizinischen Zwecken, aber auch für die Informationstechnik, bald (erfolgreich) umsetzbar sein. Das Ziel dieser Forschungen (so Oktabetz 2014: 39) , ist es, ein Maximum an Informationen auf kleinstem Raum und in einem Minimum an Zeit zu erhalten und zu verarbeiten $[\ldots]^{\text {“. }}$. Der raum- und zeitlose Informationstransport mittels verschränkter Quanten und anderer Mikroteilchen steht bereit, um neue Möglichkeiten des Informationsmanagements anzubieten, der für fast alle Lebensbereiche des Menschen einsetzbar sein wird.

Jedoch sind auch die Quantenphysik und ihr nahestehende naturwissenschaftliche Theorien dort an ihre Erklärungsgrenzen gestoßen, wo sie die in der realen Welt auftretenden Phänomene Unschärfe und Kausalität hinreichend erklären sollten. Die Logik musste in diesem Zusammenhang einspringen. Das Hinzuziehen von geisteswissenschaftlichen Erkenntnissen bleibt somit auch in modernen Informationstheorien unabdingbar.

\section{DIE ENTITÄT INFORMATION IN DER SPRACHLICHEN KOMMUNIKATION}

\subsection{Sozial- und geisteswissenschaftliche Definitionen}

Das, was wir erleben und bewirken, wird Teil des geistigen Inhalts eines universellen Speichers und kann womöglich zum Lauf der Entwicklung des Ganzen beitragen, argumentiert Oktabetz (2014) und stellt weiter fest, dass der Mensch als soziales Wesen überlebensnotwendig interagieren muss, um sich erfolgreich ins soziale Netzwerk zu 
integrieren und erfolgreich Interdependenzbeziehungen einzugehen, was jedoch ohne Informationen nicht möglich wäre. Es handelt sich dabei um von kommunizierenden Menschen beabsichtigte Informationen, um deren Austausch und Wirkung.

In der sprachlichen Welt sind Informationen Schöpfer und gleichzeitig Produkte des breiteren Welt- und Situationswissens, welches den Interpretationsraum darstellt, der aus atomaren Bedeutungen (Wörtern, Lemmata) konkrete Inhalte macht bzw. ihnen Sinn verleiht. Bei sinnvollem Informationsaustausch handelt es sich somit um den gezielten Austausch von Wirkung und Reaktion, wobei auch die Rückbeziehung erforderlich ist, was auf die unabdingbare Beziehungsherstellung zwischen Sender und Empfänger verweist (vom Bekannten wird der Zugriff auf Unbekanntes möglich).

In den späten achtziger Jahren bieten Barwise (1983) und andere (Sprach)Philosophen eine Informationsdefinition an, die neben Zeichen bzw. Impulsen auch ihre Beziehung zu jeweiligen Umwelten, zu realen Situationen der Wirklichkeit voraussetzen, was bereits auf die enge Verbundenheit von Sprache, Kognition und Informationsverarbeitung hindeutet.

Mit Carl Friedrich Weizsäcker (1988) begegnen sich im Begriff Information die Natur- und die Geisteswissenschaften. Die Information ist eigentlich Form ${ }^{1}$, kann aber nur existieren, wenn sie sich bewegt, wenn sie fließt (in Wechselwirkung steht). Für Weizsäcker (ebd.) sind unadressierte, also an keinen Empfänger gerichtete Informationen als solche nicht verwendbar. Die Brücke zur situierten Kognition (Kommunikation) (vgl. Rickheit/Strohner 1993), die aus den modernen Erklärungen des Funktionssystems Sprache nicht mehr wegzudenken ist, ist unschwer erkennbar.

\section{VERSPRACHLICHTE INFORMATIONEN}

Die Sprache verleiht Informationen Form und ermöglicht durch Unterscheidung, durch das Sich-voneinander-Abgrenzen, den Zugriff zum Erkennen und Verstehen. Sie stellt auch ein Regelwerk zur Verfügung, welches symbolisierende Verarbeitung ermöglicht, wodurch einerseits Generalisierungen und Abstrahierungen, andererseits aber auch typen- und fallbezogene Entscheidungen ermöglicht werden, was einen effizienteren und schnelleren Informationsaustausch ermöglicht. Viele Autoren teilen die Auffassung, dass zur Durchführung derartig komplexer geistiger Prozesse das dem Menschen inhärente Bewusstsein die entscheidende Rolle spiele. Dies darf jedoch bezweifelt werden, weil man für andere Lebewesen, die auch, teilweise sogar mit sprachlichen Mitteln, sicherlich aber mit anderen Kommunikationstechniken, sehr erfolgreich miteinander Informationsaustausch pflegen können (man denke dabei an (sinnvoll) sprechende Papageien, an Bienen und andere Insekten). Ist das auch ohne Bewusstsein machbar? Diese Frage wird wohl noch einige Zeit unbeantwortet bleiben.

Auf jeden Fall sind versprachlichte Informationen nicht unabhängig von Raum, Zeit und Geschwindigkeit, wie dies von den Naturwissenschaften für Informationen als solche vorausgesetzt wird, sie bedürfen nämlich nicht nur der Raumzeitlichkeit, sondern sie brauchen auch ko- und kontextuelle sowie kulturelle, natürliche und soziale Umwelten bis hin zu fachspezifischen.

1 Lat. informare ,Form geben', ,symbolisieren', ,informieren'. 


\subsection{Neurolinguistische Grundlagen der sprachlichen Kommunikation}

Weil Kommunikation in der modernen Sprachwissenschaft als Transfer konzeptueller Inhalte (Informationen) verstanden wird, wird eine enge Korrelation zwischen sprachlichen und kognitiven Prozessen angenommen. Das Gedächtnis und seine neuro-linguistischen Eigenschaften stellen die Basis bzw. die verknüpfende Instanz zwischen Sprache und Erkennen (Verstehen) dar.

Das Arbeitsgedächtnis (,Zusammenwirken zwischen dem sensorischen bzw. Ultra-Kurzzeitgedächtnis sowie dem Kurzzeit- und dem Langzeitgedächtnis') hat die Aufgabe, neue Informationen in ihre Bestandteile zu zerlegen, unbrauchbare Informationen abzublocken, nach ähnlichen Informationen unter den gespeicherten Informationen zu suchen, sie abzurufen, Neues und Altes miteinander zu verknüpfen und die durch Analyse und nachträgliche Synthese erkannten Informationen bei Bedarf kurz- oder langfristig zu speichern. Auf die gespeicherten Lemmata bezogen, muss die „Suchmaschine“ im Gedächtnis nach Synonymen suchen unter Zuhilfenahme von vorhandenem Hintergrundwissen. Auf der Ebene des Gedächtnisses kommt es durch elektrische und biochemische Vorgänge in den Nervenzellen des Kortex (den Neuronen) zum Zusammentreffen des Biologischen (z. B. synaptische Verbindungen) mit dem Rationalen und Emotionalen, mit dem Geistigen. Die Weitergabe der in der sog. Biomasse verschlüsselten Informationsteilchen (Bits) unter Zuhilfenahme von elektrischen Signalen (das neuronale Schießen), von stimulierenden und hemmenden Hormonen usw. und der anschließenden $\mathrm{Zu}-$ sammenstellung und Anknüpfung an einen oder mehrere Knotenpunkte sind jene zusammenhängende und einander bedingende Prozesse, die unser Denken und Handeln und die darauf basierende sprachliche Kommunikation ermöglichen und einen sinnvollen, im besten Fall auch sinnführenden, Informationsaustausch bereitstellen (vgl. Teržan Kopecky 2001, 2015). Dabei kommt es zu Verknüpfungen von grammatischen und semantischen Prozessen, wobei die kognitiven Prozesse durch ihre integrierende Funktion aus atomaren Bedeutungen sinnvolle Informationszusammenhänge schaffen, um zu vermeiden, dass nicht das Gesagte, sondern das Gemeinte weiter (zum Empfänger) transportiert wird (besonders wichtig im Kontext des Übersetzens und Dolmetschens).

\subsection{Kognitiver Erklärungsansatz der sprachlichen Kommunikation}

Kommunikation auf allen Ebenen bedeutet zunächst Informationsmanagement. Dieses kann von den Kommunikationsteilnehmern bewältigt werden, wenn es zur Verständigung zwischen dem zentralen Nervensystem und der Umwelt kommt. Die Umwelt wird (so die kognitive Linguistik) als ein Gefüge von natürlichen, sozialen, emotionalen, geistigen, konventionellen, rituellen, prozeduralen und ästhetischen Konstellationen verstanden. Nur in Zusammenwirken der o. a. Komponenten kommt es zur Bildung von Konzepten, was auf die unabdingbare Verbundenheit der sprachlichen Kommunikation mit der zugrunde liegenden Kultur $^{2}$ verweist (vgl. Teržan-Kopecky 2007, 2011, 2015).

2 Die Kulturkunde versteht die Kultur als eine dynamische Interaktion zwischen Normen, Codices, Wertsystemen, Gewohnheiten, Traditionen, ästhetischen Merkmalen, gestaltet vom Menschen in seinem innovativen und kreativen Auseinandersetzen mit seiner physischen, psycho-biologischen und ökologischen Umwelt. 
Jedes Lebewesen kann mit seiner Umwelt nur über Aufnahme und Weiterleitung von unterschiedlich kodierten Informationen kontaktieren. Auch von der Evolutionstheorie wird angenommen, dass nur jene Lebewesen gute (Über-)/Lebensmöglichkeiten haben, welche die Fähigkeit besitzen, Informationen am schnellsten und effektvollsten aufzunehmen und zu verarbeiten, denn nur so können sie ihr Verhalten am effizientesten anpassen - nur so können sie lernen zu überleben. Der Zugang zu Informationen bedeutet also Wissen, bedeutet Vergleich und Verknüpfung von Informationen und Schaffung von Zusammenhängen. Um diese komplexen Prozesse zu bewältigen, braucht es ein (gut) funktionierendes soziales Netzwerk, welches Interaktionen ermöglicht, wobei wir gleichzeitig veränderte, durch unsere Erfahrungswelt beeinflusste Informationen wieder in das soziale Netzwerksystem zurücksenden. Auf diese Weise konditionieren wir uns.

Kommunikation ist demzufolge eine zivilisatorische Errungenschaft schlechthin. Durch diese Auffassung wird eine Brücke geschlagen zwischen Informatik, Neurolinguistik und den kognitiven Sprachtheorien, die auf der Integration aller involvierten natürlichen Systeme aufbauen und somit für einen holistischen Zugriff zur Entität Information und ihrer Versprachlichung stehen als einer der wichtigsten Voraussetzungen für effiziente Verständigung.

\section{INFORMATIONSVERMITTLUNG IM TRANSLATORISCHEN KONTEXT}

Nach Chestermann (2000) sind Meme Zustände von aktivierten und nicht-aktivierten Synapsen im Netzwerk der im Gedächtnis agierenden Neuronen. Meme sind demnach kulturell geprägte Ideen, von denen sowohl das Denken wie auch das Übersetzen beeinflusst werden.

Meme sind Ideen, Konzepte, festgelegte Handlungsweisen, die sich wie Gene $e^{3}$ durch Vermehrung ausbreiten und von einem auf den anderen Organismus/Sprache überspringen. Sie verbreiten sich demnach durch Imitation (man vergleiche etwa das Netz wissenschaftlicher Zeitschriften SCI, wo es auch zur Verbreitung und oft Mutation von Ideen kommt). Der Mensch ist somit Quelle des Hervorbringens und Überlebens von Memen, sowie Ursache und Ziel ihrer Mutation.

Das unten Angeführte ist ein aus der Stammessprache der Adioukrou in Afrika entnommenes Beispiel für die Mem-Mutation in Richtung von lokaler Prägung bis zur allgemeineren in der christlichen Kultur vorherrschenden Konzeption/Idee (des Mems) Gott.

Gott/Nyam (der stärkste von allen Göttern), wird ursprünglich nicht als Schöpfer der Welt aufgefasst, sondern als ein erdnahes Wesen). In der Weiterverbreitung dieses Mems können wir auf der fortschreitenden Zeitachse derselben Kultur (des Stamms der Adioukrou) unter dem (Kontakt)Einfluss anderer Kulturen eine konzeptuelle

3 Die informationstragende Funktion jedes Gens wird durch seine Wechselbeziehungen zu anderen Genen und zum Ganzen gewährleistet und wird dadurch beeinflusst. Die Gene wandern von Körper zu Körper und kopieren sich selbst, aber verändern sich dadurch auch, bis zu ihrem möglichen Absterben (Dawkins 1976). 
Verschiebung beobachten. Der Gott Nyam wird nämlich im Laufe der Zeit in den Himmel gehoben, jedoch bleibt er noch eine Zeit lang mit der Göttin Erde verheiratet und ist keine sonderlich starke moralische Entität. Später jedoch mutiert er in einen Gott, der die Welt erschaffen hat, der sich um Menschen kümmert und tugendhaft ist. Damit nähert er sich der Konzeption des christlichen Glaubens und des christlichen Gottes. Dieser Weg der Anpassung und Veränderung eines Konzeptes beleuchtet sehr einprägsam die Theorie der Mem-Mutationen durch den (inter)kulturellen Kontakt im Verlauf der Geschichte.

\section{FACHSPRACHE ALS MONOSEMIERENDE KOMMUNIKATIONSUM- WELT}

Die (fachbezogene) Kommunikation ist im Prinzip eine durch fachliche Kontexte monosemierte Kommunikation mit ihren universellen Merkmalen (z. B. Kohärenz, Kohäsion, Annehmbarkeit, Informationsbezogenheit, Intertextualität usw.), die sich zusätzlich mit spezifischen fachbezogenen Eigenschaften verbinden.

Ein Fachtext ist eine in sich verbundene, entsprechend der Fachlogik strukturierte und abgeschlossene komplexe sprachliche Erscheinungsform. Er bezieht sich auf einen für eine gewisse fachliche Tätigkeit typischen Tatbestand. Im Fachtext gebraucht der Textproduzent der Situation und dem Kommunikationsziel entsprechende sprachliche und außersprachliche Ausdrucksmittel (auch visuelle und andere nichtsprachliche Ausdrucksmittel können eingesetzt werden, wie etwa Filme, Fotografien, Zeichnungen, Symbole, Formeln, Gleichungen, Tabellen usw.) (vgl. Prunč 2007, Ostapenko 2007).

Fachliche und kulturspezifische Einflüsse greifen bei Fachtexten stark ineinander. Auch Fachdiskurse sind nämlich geografisch, national, sozial und fachlich an engere Gruppierungen von Fachdiskursteilnehmern gebunden. Als solche sind sie aber in einem größeren Kontinuum verwurzelt, nämlich dem der Kultur als jenem globalen Verhaltensmuster, aus welchem auch (fach)spezifische Verhaltensmuster ableitbar sind. Veranschaulicht sei diese Behauptung durch die Unterschiede in der Konzeption von rechtlichen Termini, die jeweils unterschiedlichen Kultur- und Rechtssystemen zuzuordnen sind. Das angloamerikanische Recht (common-law) verursacht durch seine unterschiedliche kulturelle Provenienz gegenüber dem kontinentalen Recht (beide sind (fachlich)kulturelle Normensysteme) kognitive Hindernisse.

Von der modernen Definition der Begriffe Fachtext und Fachkommunikation wird eine binäre Teilung auf Fach- und Nicht-Fachtexte und deren Übersetzungen aberkannt. Vielmehr wird von einem Kontinuum ausgegangen, innerhalb dessen jeder Text durch eine größere oder geringere Fachlichkeit ausgezeichnet wird.

\subsection{Werbesprache}

Auch ästhetische und emotionale Komponenten spielen im fachlichen Diskurs eine Rolle. Fachtexte müssen nämlich auch überzeugen, gefallen, Interesse wecken, warnen, an die menschlichen Sinne appellieren usw. Dies schaffen sie natürlich umso effektvoller, je mehr sie auf positive (mitunter auch negative) Emotionen zurückgreifen und durch ästhetische Merkmale gefallen. Besonders starke emotionale und/oder 
ästhetische Prägung weisen Fachtexte wie Werbetexte, politische Reden, PR-Tätigkeiten usw. auf (vgl. Prunč 2007, Teržan-Kopecky 2007).

Premrov (2016) behandelte in ihrer Dissertation die kommunikativen Besonderheiten der Werbesprache in der TV-Werbung, womit wir die o. a. Behauptungen zu kulturellen sowie ästhetischen und emotionalen Komponenten von Fachtexten veranschaulichen möchten. Premrov richtete ihr Interesse auf die von unterschiedlichen Rezeptoren ausgelösten Impulse/Informationen: Das sind akustische und optische sowie filmische Elemente und der Einfluss von Farben und von Musik auf die Erregung von unterschiedlichen Typen von Emotionen in Relation zu ihrer Beeinflussung der Informationsaufnahme seitens des Zielpublikums. Die kommunikativen Effekte der Werbung wurden auch im Hinblick auf die Sender und Empfänger ausgewertet: Die Probanden bekamen optische (Bild, Film), akustische (Musik und Sprache) und kombinierte Reize vorgeführt. Auf diese Weise - durch Befragung der Probanden und durch gemessene physiologische Reaktionen - wurden Bedeutung und Funktion der durch Sprache sowie durch Bild und Ton ausgelösten emotionalen Schemata und der Einfluss von Emotionen auf das Gedächtnis ermittelt. Dadurch kamen die Verbindungen zwischen Sprache und Emotionen und deren Auslösern in Abhängigkeit vom kulturellen Hintergrund der Empfänger und von ihrem natürlichen Geschlecht an den Tag. Eine weitere Variable war die Aufgliederung der Probanden auf slowenische und deutsche Muttersprachler, wobei die Aufmerksamkeit auf jeweiligen emotionalen Reaktionsmustern und ihrer Wechselwirkung mit dem Kaufverhalten lag. Dabei stellte sich heraus, dass sowohl bei slowenischen als auch bei deutschen Sprechern die nichtsprachlichen Reize in Bezug auf die Effizienz der ausgelösten emotionalen Reaktionen deutlich überwiegen, wobei aber die sprachlichen Reize/Informationen doch auch in der erwähnten Hinsicht erfolgreicher waren als die kombinierten Reizauslöser (Wort und Bild; Wort und Ton), denn die Probanden reagierten intensiver auf die sprachlich kodierten Informationen als auf Ton oder Bild. Dabei handelt es sich offensichtlich um ein universelles kultur-, sprachen- und geschlechtsunabhängiges Phänomen. Es zeigten sich aber Unterschiede in der Aktivierung des Emotionspotentials in Korrelation zum Geschlecht der Probanden, was darauf hindeutet, dass die Unterschiede im emotionalen und dem darauf basierenden sprachlichen Verhalten in gewissem Maße doch auch geschlechtsbezogen sind.

Die Ergebnisse der oben vorgestellten Studie bestätigen die bereits in unterschiedlichen anderen themenbezogenen Untersuchungen wahrgenommenen Beobachtungen, dass die Einbettung eines jeglichen sprachlichen Produktes in sein kulturelles/soziales System mit der gleichzeitigen Bündelung unterschiedlicher Reize zur erfolgreichen Informationsvermittlung enorm viel beitragen kann, vor allem, wenn es sich um emotionale Inhalte mit ästhetischer Prägung handelt.

\subsection{Juristische Texte}

Die terminologische Unangepasstheit (das Fehlen einer inter- und transnationalen Fachbegriffskoordinierung) erzeugt mitunter massive kognitive und kommunikative Hürden. Auch Fachdiskurse sind demnach geografisch, national, sozial an engere Gruppierungen von Fachdiskursteilnehmern gebunden. Als solche sind sie aber in 
einem größeren Kontinuum verwurzelt, nämlich dem der Kultur als jenem globalen Verhaltensmuster, aus welchem auch (fach)spezifische Verhaltensmuster ableitbar sind. Nach wie vor überspielen sie den übernationalen Drang nach Vereinheitlichung (Best/Kalina 2008; Ostapenko 2007).

Veranschaulicht sei diese Behauptung durch die kultur- und folglich auch fachdiskursbedingten Unterschiede in der Konzeption von rechtlichen Termini, die jeweils unterschiedlichen Kultur- und Rechtssystemen zuzuordnen sind. Das angloamerikanische Recht (common-law) verursacht durch seine unterschiedliche kulturelle Provenienz gegenüber dem kontinentalen Recht (beide sind (fachlich)kulturelle Normensysteme) terminologische Probleme beim Übersetzen von juristischen Fachtexten:

a court, tribunal-dt. Gericht, slow. sodišče (im Slow. und Dt. jeweils nur ein Terminus)

b Obligationsrecht/obligacijsko pravo (im Engl. keine direkte Entsprechung)

c equity (,Gerechtigkeitsrecht) (im Dt. keine direkte Entsprechung)

d board of directors (,Verwaltungsausschuss') (im Slow. wie auch im Dt. keine direkte Entsprechung)

Die obigen Beispiele zeigen kulturbedingte Differenzen im terminologischen Bereich zwischen dem angloamerikanischen und dem kontinentalen Recht, bedingt durch unterschiedliche Auffassungen und kognitive Modelle der jeweiligen Gerichtsbarkeit, die kulturell aus Denk- und Handlungsmustern abgeleitet wurden (Kocbek 2010). Übersetzer und Dolmetscher müssen lexikalische Leerstellen durch Umschreibungen und andere Techniken überbrücken. Das führt aber zur gegenseitigen Beeinflussung (bzw. Mem-Mutation).

Dass der Einfluss von Rechtstexten auf die slowenischen Entsprechungen im Bereich der Rechtsprechung seitens der „gebenden“ Kultur ausgesprochen groß war, zeigte sich vor allem beim Studium von Übersetzungen des Kaiserlich-Königlichen Reichsgesetzes und anderer lokaler Gesetze der K.-u.-k.-Monarchie. Die übersetzten Rechtstexte wurden nämlich in eine andere Raumzeitlichkeit (in den slowenischen Kulturraum) versetzt, wodurch neue Meme gestaltet wurden.

In der Karwoche findet nur ein Wochenmarkt u. zw. am Gründonnerstag statt > Če pade tedenski tržni dan na praznik, se isti vrši dan poprej.

Die unmittelbare Aufsicht über den Markt übt die Marktpolizei. [...] > Prodajalci in kupci so dolžni pokoravati se odredbam tržnih organov $v$ okviru tega pravilnika $[\ldots]^{4}$

4 Die o. a. Beispiele stammen aus der „Gemeindeordnung der Stadt Marburg “(1872), die im Rahmen des Forschungsprojektes (2004-2007) ,Slowenische Übersetzungen deutscher Texte in der Periode der österreichisch-ungarischen Monarchie" (Projektleiter: Prof. Dr. Karmen Teržan Kopecky, Universität Maribor, und Prof. Dr. Erich Prunč, Karl-Franzens-Universität Graz) untersucht wurde. 
Kultur- und traditionsbedingt konnten einige typische Züge von Fachtermini bei ihrer Übersetzung ins Slowenische beobachtet werden. Zusammenfasend kann man feststellen, dass die slowenischen Übersetzer zumeist die konkrete Benennung von kirchlichen Feiertagen und/oder Gepflogenheiten zu den christlichen Feiertagen vermeiden, indem sie z. B. einen konkreten Feiertag zum allgemeineren Fest machen, oder die Marktpolizei zu »Marktorganen« verallgemeinern, um an besonders kultur- oder systemgebundenen Stellen eine translatorische Distanz zu schaffen, gleichzeitig aber den Kulturtransfer zu vollziehen.

\subsection{Literatursprache}

Die u. a. Beispiele aus Volksbüchern aus dem Korpus deutsch-slowenischer Übersetzungen, entnommen aus dem Werk Legenden vom Rübezahl (1842), sprechen eine deutliche Sprache:

a i werd schon dem Schurrer a Manier lernen” > Grešnika bom pa že naučil kozje molitvice

b so soll der Pertl wartn, bis der Quarzhirn stirbt, dann wird Hochzeit > Pavel bo že počakal, da se stari jeklen stegne, potem bode pa ženitovanje

c [...] Ja Gabi, ich hab Dir auch a Christkind mitbracht. [...] Franica, tudi nekaj za potico sem ti prinesla.

Die kulturellen Unterschiede schlagen sich im Gebrauch neuer, zielsprachenspezifischer Meme nieder. Vor allem die Phraseme müssen natürlich kulturspezifisch neu „erfunden“ werden (Beispiel a). Raue und besonders starke Ausdrücke werden üblicherweise in slowenischen Übersetzungen abgeschwächt. Ein seltenes Gegenteil finden wir im Beispiel c, wo in der Zielsprache sogar ein „,derberer“ Ausdruck verwendet wurde (sterben wird zu stegniti se). Auffällig ist beim letzten Beispiel die bereits oben erwähnte Neigung der Übersetzer ins Slowenische zur Neutralisierung von Feiertagsnamen. So wird im obigen Zitat a Christkind zu potica.

Als Veranschaulichung der äußersten Sensibilität bei der Wahl der Versprachlichung der zu vermittelnden Konzepte (Ideen, Gefühle) in künstlerischen Texten moderner literarischer (Informations)Welten, begleitet von ästhetischen, sehr oft aber auch ethischen Begriffskonstellationen, sei hier der Titel der Fabel von La Fontaine $L a$ Cigale et la Fourmi (1668) angeführt, welcher, um sowohl inhaltlich als auch ästhetisch entsprechend übersetzt werden zu können, nicht allein mit semantischem Transfer dem Original gerecht wird. Es musste noch auf die symbolisierende Kraft der Grammatik zurückgegriffen werden, wodurch propositionelle, aber auch logische Relationen zwischen Pupille und Delle in ihren feinsten Ausprägungen den Übersetzern zur Verfügung gestellt wurden.

a Die Zikade und die Ameise $\rightarrow$ Škržat in mravlja (Zielsprache Slowenisch)

b Cvrček in mravlja $\rightarrow$ Grille und die Ameise (Zielsprache Deutsch)

c Cvrček in mravlja $\rightarrow$ Der Grashüpfer und die Ameise (Zielsprache Deutsch) 
Die o. a. Beispiele zeigen in ihrer Einzigartigkeit, wie alle Verbalisierungskomponenten, sogar die ,als nüchtern“ empfundene Grammatik, poetische Lieblichkeit oder das Gegenteil dazu zu vermitteln vermögen. Die Information an sich (die Idee) bleibt dabei eine Konstante, die sprachempfindlichen/kultursensiblen Variablen tragen der Poetik Rechnung.

Das Sem Leichtigkeit (Femininum) konnte durch semantische, morphologische und pragmatische Analyse eruiert werden. Das macht die Entscheidung des Übersetzers für die Zikade im Deutschen und kobilica im Slowenischen nach Auffassung von Ana Serena Papa (2009) zur besten Wahl, da das grammatische Geschlecht $\mathrm{Fe}$ mininum in diesem Fall das Sem der Leichtigkeit gewährleistet, was maskuline Tiere, auch wenn sie klein und freundlich sind, nicht hervorzurufen in der Lage sind. Das ist ein schönes Beispiel für die zur Sinngestaltung notwendige, zunächst recht allgemeine Information.

\section{Die Stelle, wo mein Nußbaum blüht, liegt vierzehn Tage hinterm Glücke [...] (Christine Lavant, Spindel im Mond)}

Um das von der Autorin intendierte Informationspotenzial der Aussage des oben angeführten Verses »entschlüsseln« zu können, ist ein gebündeltes Wissenspotenzial erforderlich, z. B. über die sprachliche Kompetenz im Allgemeinen, über die stilistischen Merkmale der literarischen Sprache, über die Kanonisierungshinweise zum besagten Werk, bis hin zum Spezialwissen über das Leben der Autorin, über ihren literarischen Werdegang, über ihre Sorgen und Nöte. Nur so kann der anfangs große Interpretationsspielraum eingeengt werden.

\section{ZUSAMMENFASSENDE GEDANKEN}

Folgendes sollte in dem oben Dargelegten mitgeteilt werden: Informationen dienen der Vergrößerung und Erneuerung des Wissens. Neuere Wissenschaftszweige (z. B. Bioinformatik) gehen davon aus, dass Gene als Informationsüberträger den Bauplan des Lebens in sich tragen. Die Genome als vollständige Informationen bestimmen die charakteristischen Eigenschaften eines jeden Lebewesens.

Sobald Informationen mit spezifischen Kulturen in Berührung kommen, werden sie kodiert (sie nehmen die Form unterschiedlicher Zeichen an). Die komplexeste Form ist eben Sprache, die durch ihre symbolisierende und metaphorisierende Kraft eine enorme Anzahl an Referenzen herzustellen vermag und dadurch Kommunikation ermöglicht. Die Einbeziehung von sprachlichen und außersprachlichen Umwelten zu Kommunikationszwecken ist unabdingbar. Bedeutungen entstehen durch Verknüpfung zwischen Information und Informationsquelle, durch Zerlegungen in Einzelteilchen (Bits) mit anschließender Zusammenfassung von Eigenschaften und/oder Generierung neuer Informationen aus den Vorhandenen (Teržan-Kopecky 2007, 2011).

Auf diese Weise wird Information zu Kommunikationszwecken zu Wort, womit sie Form erhält und abgerufen/(weiter)vermittelt werden kann. Worte sind jedoch durch Begriffsoffenheit, Unschärfe und Interpretationsvielfalt gekennzeichnet. Das zeigt 
sich mitunter auch in der Tatsache, dass z. B. in Phrasemen eine Begriffseinheit die Grenzen des einzelnen Wortes übersteigt oder umgekehrt einzelne Wortgrenzen mehrere Begriffe umfassen (z. B. Komposita). Stabiler ist dagegen die Silbe (vgl. Wurzel 1984). Weitgehend monosemiert und zu Verständigungszwecken geeignet wird der vage Begriff Wort erst in konkreten Kommunikationsakten, die ihrerseits generations-, geschlechts-, fachbestimmt sind und jeweils von eigenem Erfahrungspotenzial geprägt werden. Auf diese Weise erhalten Wort und Sprache eindeutig zuzuordnende Begriffe, die ihrerseits, verpflanzt in andere Kulturen, neue Begriffswelten generieren können. Die einzelsprachspezifische Ebene weist Besonderheiten jeder Einzelsprache auf der mikrokulturellen Ebene auf. Das ist jener Bereich der Sprache, der am sensibelsten die jeweils aktuellen Kulturmuster aufgreift. Hier lebt die Sprache unmittelbar mit den Gewohnheiten, Bräuchen und Mustern der sozialen, kulturellen und religiösen Welt einer jeden Sprachgemeinschaft zusammen. Somit ist klar, dass Sprache, auch Fachsprache, und Kultur ein dynamisches Kontinuum bilden, was aus den oben gezeigten Beispielen eindeutig hervorgeht. Für die Übersetzungstheorie und Praxis ist die Mem-Idee von besonderer Bedeutung. Meme stellen Einheiten standardisierter Verhaltensweisen und gesellschaftlich-kultureller Kategorien dar. In ihrer Wanderung durch Zeit und Raum passen sich Meme neuen Gegebenheiten an (sie mutieren). Diese Prozesse werden massiv durch die translatorische Tätigkeit gefördert.

Im oben angeführten Zusammenhang sind die Worte Bakhtins (1934/35: 193), mit denen er bereits in den dreißiger Jahren des letzten Jahrhunderts über die Verbindung von Kultur und Sprache mit ihrer Umwelt nachdachte, bezeichnend:

All words have a "taste" of a profession, a genre, a tendency, an age group, the day an hour. Each word tastes of the context and contexts in which it has lived its socially charged life, all words and forms are populated by intentions.

Die Erkenntnisse der vorgenannten modernen Wissenschaften (allen voran der Quantenphysik, der Mikrobiologe und der Informationstechnologie) ermöglichen neue Einsichten in die Beschaffenheit der Entität Information außerhalb ihrer sprachlichen Existenz, stellen aber auch ein enormes Potential für die Sprachforschung bereit und laden zu zukünftigen komplementären Untersuchungen ein.

\section{Literatur}

BAKHTIN, Mikhail Mikhailovich (1981 [1934/35]) „Discourse in the Novel.” In: M. Holquist (Hrsg.), The dialogic imagination. Übers. von C. Emerson/M. Holquist. Austin/London: University of Texas press, 259-422.

BARWISE, Jon (1983) The Situation in Logic. Stanford: Center for the Study of Language and Information.

BEST, Joanna/Silvia KALINA (2002) Übersetzen und Dolmetschen. Tübingen/ Basel: A. Francke Verlag.

CHESTERMAN, Andrew (2000) Memes of Translation. Amsterdam/Philadelphia: John Benjamins. 
DAWKINS, Richard (1976) The Selfish Gene. Oxford: Oxford University Press.

FISCHER, Kerstin (2000) From Cognitive Semantics to Lexical Pragmatics. Berlin/ New York: de Gruyter Mouton.

„Gemeindeordnung der Stadt Marburg“ (1872). Landesgesetz- und Verordnungsblatt für das Herzogthum Steiermark.

KOCBEK, Alenka (2010) Načelo kulturne vpetosti jezika v prevodih pravnih besedil (na primeru gospodarske pogodbe kot besedilne vrste). Doktorska disertacija. Ljubljana: Univerza v Ljubljani, Filozofska fakulteta.

LA FONTAINE (1668) La Cigale et la Fourmi. Paris: C. Barbin.

LANGACKER, Wayne Roland (1999) Grammar and Conceptualisation. Berlin/New York: de Gruyter Mouton.

LAVANT, Christine (1959) Spindel im Mond. Gedichte. Salzburg: Otto Müller.

MUSÄUS, Johann Karl (1842) Legenden vom Rübezahl. München: Winkler Verlag.

OKTABETZ, Kurt (2014) Das Infoversum. Graz: Leykam.

OSTAPENKO, Valentyna (2007) Zu Fachtextsorten. Vernetzung von Fachtextsorten.

Textsorten der Normung in der technischen Harmonisierung. Berlin: Frank\&Timme.

PAPA, Ana Serena (2009) Vloga kognitivne besedne semantike za človeka kot misleče bitje in prevajalca./The Role of Cognitive Word Semantics for the Individual as a Thinking Human Being). Diplomsko delo. Maribor: Univerza v Mariboru, Filozofska fakulteta.

PREMROV, Emira (2016) Vpliv jezikovnih dražljajev televizijskih oglasov na čustveno odzivanje z vključitvijo meritve fizioloških parametrov. Doktorska disertacija. Maribor: Univerza v Mariboru, Filozofska fakulteta.

PRUNČ, Erich (2007) Entwicklungslinien der Translationswissenschaft. Von den Asymmetrien der Sprachen zu den Asymmetrien der Macht. Berlin: Frank \& Timme.

RICKHEIT, Gert/Hans STOHNER (1993) Grundlagen der kognitiven Sprachverarbeitung. Tübingen/Basel: A. Francke Verlag.

TERŽAN-KOPECKY, Karmen (2001) Psihološke dimenzije jezikovnih ravnanj. Maribor: Pedagoška fakulteta.

TERŽAN-KOPECKY, Karmen (2007) „Utrinki iz zakladnice slovenskih prevodov nemških besedil v raznolikosti njihovih medsebojnih odnosov." In: ead. (Hrsg.), Slovenski prevodi nemških besedil v obdobju avstro-ogrske monarhije: znanstvene refleksije. Maribor: Filozofska fakulteta, 73-88.

TERŽAN-KOPECKY, Karmen (2011) „Sprachlich übergreifende Fachkommunikation als kulturelles Phänomen vor dem Hintergrund der Kognition.“ In: M. Hahn/G. Wazel (Hrsg.), Theorie und Praxis des DaF- und DaZ-Unterrichts heute: 20 Jahre Institut für Interkulturelle Kommunikation e. V.. Frankfurt a. Main [etc.]: Peter Lang, 289-307.

TERŽAN-KOPECKY, Karmen (2015) „Natürlichkeitstheorie und kognitive Hintergründe des Zweitspracherwerbs oder „Mm, to je lecker““، In: U Valenčič Arh/D. Čuden (Hrsg.), V labirintu jezika /Im Labyrinth der Sprache. Ljubljana: Znanstvena založba Filozofske fakultete, 363-373.

WEIZSÄCKER, Carl Friedrich (1988) Aufbau der Physik. München: DTV. 
WIENER, Norbert (1948) Cybernetics: Or Control and Communication in the Animal and the Machine. Paris: Hermann/Cambridge: Technology press/New York: J. Wiley \& Sons.

WURZEL, Wolfgang Ullrich (1984) Flexionsmorphologie und Natürlichkeit. Berlin: Akademie Verlag.

\section{Zusammenfassung \\ DIE ENTITÄT INFORMATION IM SPANNUNGSFELD ZWISCHEN WORT UND TEXT}

Dass die Information eine selbstständige Elementarform ist, die jegliche Lebensformen bedingt, ist eine der zentralen Aussagen zum Thema Information, die vom Bereich der Quantenphysik und der ihr nahestehenden Naturwissenschaften zur Forschung nach weiteren Erkenntnissen zur Verfügung gestellt wurde. Die Quantenphysik stellt neues Wissen bereit, wonach die Informationsübertragung bislang ungeahnte Geschwindigkeiten überwinden kann, was die Verschiebung von lokalem zu globalem Handeln auch auf dem Bereich der Forschung ermöglichte.

Diese naturwissenschaftlichen Erkenntnisse werden im gegenwärtigen Beitrag mit der kommunikativen Effizienz von versprachlichten Informationen in Verbindung gebracht. Informationen existieren auf allen Ebenen (vom Universum bis zu den kleinsten Zellteichen). Beobachtet von der Warte ihrer sprachlichen Existenz aus, zeigen sich sowohl universelle, gleichzeitig aber auch prototypische, sprach- und kultureigene bis hin zu fachbezogenen und der Erfahrungswelt eines jeden Individuums entspringenden System-Eigenschaften. Im Wesentlichen unterliegt das natürliche System Sprache ähnlichen Ordnungs- und Funktionsmechanismen wie andere lebende Systeme.

Ein zweckdienlicher Informationsaustausch unterliegt demnach zahlreichen Bedingungen und Einflüssen, die vom sprachlichen System als solchem ausgehen (z. B. Grammatik, Semiotik, Semantik, Pragmatik usw.), um sich mit möglichst vielen Informationsquellen aus der Innen- und der Außenwelt der Kommunikatoren zu einer sinnvollen Gesamtheit zusammenzuschließen. Informationen sind nur in ihren sozialen und natürlichen Umwelten für Kommunikationsteilnehmer sinnvoll einsetzbar.

Der Beschaffenheit dieser Prozesse wird im vorliegenden Aufsatz mit Berücksichtigung unterschiedlicher Arten des Informationsaustausches (fachsprachliche, literarische, inter- und transkulturelle Kontexte) nachgegangen.

Schlüsselwörter: Information, Informationstechnologie, Kognition, Kommunikation, Translation 


\section{Abstract \\ ENTITY INFORMATION IN THE TENSION FIELD BETWEEN WORD AND TEXT}

Information is in itself an elementary form, which affects all aspects of life; this paper seeks to shed light on how the insights of quantum-physics and other related natural sciences can help us deepen our understanding of information. Quantum physics has given us an insight into the unimaginable speed of information transfer. New scientific achievements involving a shift from local to global thinking and researching was made possible, which effects also the quality of studies of language-coded information. The latter exists on all levels and spheres (from space to the smallest cell particles).

If the latter is observed from the point of view of language, a universal but at the same time also prototypical and idiosyncratically originated system properties are the basic sources of meaning and sense, transported by language. Cultural experience, business and other professional activities and meanings are constituents of communicative situations of various text types as well.

Language is basically a natural system and as such based on similar functional mechanisms as other living systems. A goal-oriented information exchange depends on numerous conditions and influences of the language system as such (e.g. grammar, semiotics, semantics, pragmatics etc.) in order to connect itself with as many information sources as possible from the inner- and outer world of communicators to create a reasonable entirety.

Information can provide communicative meaning, only if it is used in social and natural environments. The nature of these processes is presented in this paper with regard to different kinds of information exchange.

Keywords: information, information-technology, cognition, communication, translation

\section{Povzetek \\ ENTITETA INFORMACIJA V POLJU NAPETOSTI MED BESEDO IN BESEDILOM}

Informacija je samostojna elementarna entiteta, ki pogojuje vse oblike življenja, kar je tudi eno od osnovnih izhodišč obravnave pojma informacija. Opredelitev v članku je prevzeta iz področij kvantne fizike in njej sorodnih naravoslovnih ved in ponuja nove možnosti raziskovanja tega pojava, ki ga pričujoči prispevek poveže s komunikacijskim učinkom ubesedenih informacij. Spoznanja kvantne fizike prinašajo nova spoznanja o doslej neslutenih hitrostih prenašanja informacij, tudi na najdaljše razdalje, kar je omogočilo premik od lokalnih h globalnim raziskavam. Informacije namreč obstajajo na vseh nivojih (od vesolja do najmanjših delčkov celice).

Kadar obravnavamo informacije z vidika njihove jezikovne pojavnosti, opažamo univerzalne, hkrati pa tudi prototipične ter jezikovno- in kulturno izzvane sistemske 
lastnosti, ki segajo vse do izkustvenih svetov različnih strokovnih področij. V svojem bistvu podlega naravni sistem jezik primerljivim urejevalnim mehanizmom, kot to velja za druge žive sisteme.

Smiselna in ciljno usmerjena izmenjava informacij je odvisna od številnih pogojev in vplivov, ki izvirajo iz jezikovnega sistema (npr. slovnica, semiotika, semantika, pragmatika itd.), da bi se slednji povezal s kar največjim številom informacijskih virov iz zunanjega in notranjega sveta udeležencev komunikacije in na ta način tvoril smiselno celoto.

Ključne besede: informacija, informacijska tehnologija, kognicija, komunikacija, prevajanje 